\title{
Learning Processes in Matching and Oddity: The Oddity Preference Effect and Sample Reinforcement
}

\author{
Anthony A. Wright \\ University of Texas Medical School at Houston \\ Juan D. Delius \\ University of Konstanz

\begin{abstract}
Eight pigeons learned either matching (to sample) or oddity (from sample) with or without reward for sample responding. The training stimuli were coarse-white, fine-black, or smooth-mauve gravels in pots with buried grain as the reinforcer. Oddity without sample reward was learned most rapidly, followed by matching with sample reward, oddity with sample reward, and matching without sample reward. Transfer was related to acquisition rate: The oddity group without sample reward showed full (equal to baseline) color and texture transfer; the matching group with sample reward showed partial texture transfer; other groups showed no transfer. Sample reward was shown to determine rate of acquisition of matching and oddity and the oddity preference effect. The results are discussed in terms of item-specific associations operating early in learning prior to any relational learning between sample and comparison stimuli.
\end{abstract}

Keywords: matching to sample, oddity from sample, oddity preference effect, concept learning, pigeons

The oddity-preference effect (OPE), preference for a stimulus that does not match the sample, has puzzled researchers for nearly a half century (Ginsburg, 1957). The OPE has been shown for a variety of species such as apes (Davenport \& Menzel, 1960), monkeys (e.g., Mishkin \& Delacour, 1975), and jays (Wilson, Mackintosh, \& Boakes, 1985b), with the greatest focus being on pigeons (e.g., Berryman, Cumming, Cohen, \& Johnson, 1965; Cumming \& Berryman, 1965; Ginsburg, 1957; Wilson et al., 1985b; Zentall, Edwards, Moore, \& Hogan, 1981). Pigeons trained on oddity, for example, show an OPE by learning more rapidly than those trained on matching. Also, matching performance may begin below chance $(50 \%$ correct) and oddity above chance (e.g., Cumming \& Berryman, 1965; Wilson et al., 1985b; Zentall et al., 1981), but this latter finding may be obscured by averaging over a substantial number of trials (e.g., 64 to 120 trials). Wilson et al. (1985b) concluded that pigeons have a "marked preference for oddity" and that this oddity preference persists throughout training and even "somehow interferes with [their] ability to display transfer of the matching or oddity rule" (p. 324). They also acknowledged that despite this "marked preference for oddity" the cause of the OPE "is not understood" (Wilson, Mackintosh, \& Boakes, 1985a, p. 302). Strangely, there have been no attempts to discover what is responsible for the OPE in the 20 years since the Wilson et al. articles. The lack of attempts to discover what causes the

Anthony A. Wright, Department of Neurobiology and Anatomy, University of Texas Medical School at Houston; Juan D. Delius, Allgemeine Psychologie, University of Konstanz, Konstanz, Germany.

This research was supported by National Institute of Mental Health Grants MH-35202 and MH-61798 to Anthony A. Wright. We thank Jacquelyne J. Rivera for her careful conduct of the experiments and Jeffrey S. Katz for helpful comments on a draft of this article.

Correspondence concerning this article should be addressed to Anthony A. Wright, Department of Neurobiology and Anatomy, University of Texas Medical School at Houston, P.O. Box 20708, Houston, TX 77225. E-mail: anthony.a.wright@uth.tmc.edu
OPE may have come from a general acceptance that the OPE is somehow predetermined-an "initial preference for the odd stimulus" (Wilson et al., 1985a, p. 308).

Despite claims that pigeons come to the experimental setting predisposed with an oddity preference, the position taken in this article is that there are overlooked aspects of the experimental procedure that contribute to producing the OPE. The focus in this article is on how the lack of reinforcement for sample responding contributes to producing the OPE in pigeons. This is not the first time that the lack of sample reward has been proposed to cause the OPE. Such a proposal was made some 4 decades ago (Berryman et al., 1965; Cumming \& Berryman, 1965) and has been repeated by others in the interim (e.g., Carter \& Werner, 1978; Delius, 1994). Strangely, since no one has previously tested this proposal, one can only guess that its common-sense appeal has kept it viable all these years. To see how the lack of reinforcement for sample responding might be a factor in producing the OPE, consider a typical matching or oddity trial. Pecking the sample stimulus produces two choice or comparison stimuli; one stimulus is the same and the other is different from the sample. No reinforcement (extinction) for sample responding presumably weakens the tendency to choose the same stimulus (i.e., matching comparison) and increases the likelihood to choose the nonmatching (oddity) comparison. The opposite tendency or bias will presumably be created when sample responding is rewarded. These biases or preferences (e.g., OPE) will be shown to be influenced by whether or not sample responding is rewarded (henceforth referred to as sample reward).

The procedure used in these studies was a gravel-digging task that was originally based upon pigeons foraging in sandy lots for seeds (Siemann, Delius, \& Wright, 1996; Wright \& Delius, 1994). In these experiments, pigeons dug in small ceramic pots filled with different gravels. On each trial, three pots of gravel (sample in center) were presented on a tray outside the cage. The clear cover over the sample pot was opened and the pigeon dug in the gravel. As it was eating the second of three seeds buried in the sample pot, 
covers over both side pots were opened simultaneously. Pigeons trained on matching were rewarded with a cache of buried seeds in the matching gravel pot and those on oddity with seeds in the nonmatching gravel pot. They learned these tasks in less than 32 trials (8 trials per day). Neither group showed an OPE, but the rapid learning and/or small sample reward may have masked any OPE. In the present study, we used three training stimuli to slow acquisition. The matching and oddity groups were each divided into two subgroups receiving either a large sample reward or no sample reward.

The gravel-digging task differs from key-pecking tasks in that pigeons manipulate and change the gravel stimulus as they clear gravel out of the pots. Moreover, pigeons reveal buried seeds themselves rather than have the reward simply presented to them. When they made errors, they often dug to the bottom of the incorrect gravel (possibly emphasizing a mistake) and then were left with that same gravel mess on a subsequent correction trial. Eventually, the incorrect pot would contain no gravel, prompting the pigeon to switch to the correct pot.

\section{Experiment 1}

\section{Method}

\section{Subjects}

The subjects were 8 experimentally naive White Carneaux (Columba livia) pigeons, 6 to 7 years old, from the Palmetto Pigeon Plant (Sumter, SC). They were maintained on a 14:10-hr light-dark cycle with water and grit continuously available in their home cage in a state-approved vivarium. Daily experimental sessions were conducted 5 days each week if the pigeons were within $82 \%$ to $88 \%$ of their free-feeding weights.

\section{Apparatus}

The testing apparatus was the same clear Plexiglas tray, ceramic pots, and basic procedures used in the Wright and Delius (1994) study. The tray was hooked to the front of the pigeon's cage and held three gravel pots (5.4- $\mathrm{cm}$ diameter at top, $4.8-\mathrm{cm}$ diameter at bottom, and $3.0-\mathrm{cm}$ high) in separate compartments; the pots had separate clear, sliding lids. Two of the three training stimuli were a coarse white broken-coral gravel (mean diameter of $0.64 \mathrm{~cm}$ ) and a fine black volcanic gravel (mean diameter of $0.32 \mathrm{~cm}$ ) used by Wright and Delius; the third stimulus was a shinier, smoother mauve Ultrastone gravel (mean diameter of $0.51 \mathrm{~cm}$ ). The gravel depth was $1.2 \mathrm{~cm}$ controlled by casting resin at the bottom of the pot and by scraping off excess gravel in the pot with a straight edge.

\section{Procedure}

Experimental sessions were conducted with pairs of pigeons moved to a separate room. Trials alternated with intertrial intervals equal to the other pigeon's trial time.

Pretraining. There were four pretraining sessions with eight diggingtraining trials per session. Seeds (one each of popcorn, white pea, wheat, milo, black pea) were buried successively deeper in the gravel. The type of training gravel (one of three) and the right or left location varied quasirandomly with no pretraining given in the center (sample) position.

Training. Groups were trained on either matching-to-sample or oddityfrom-sample, and half of each group was given sample reward and the other half no sample reward. Pigeons receiving sample reward found 20 seeds buried at the bottom of each sample pot: 1 kernel corn, 1 popcorn, 1 black pea, 2 white peas, 3 kafir, 3 millet, 4 milo, and 5 wheat. A trial began by opening the clear Plexiglas sliding cover over the sample and allowing the pigeon to dig in that pot. Doors to the side or comparison pots were opened (simultaneously) when the pigeon was finishing eating the 20 seeds in the sample pot so that it would be centered when covers to the two side pots were opened. (Side-pot covers were joined and made from a single piece of Plexiglas.)

Pigeons not receiving sample reward required some sample-digging training on the first two sessions so that they would be digging in the sample pot when the side covers were opened. One bird of each testing pair received sample reward, and the other did not. Digging time of the sample-without-reward bird was yoked to the sample digging time of the sample-with-reward bird on a trial-by-trial basis from the third session (except on transfer test trials).

Following their side-pot choice (gravel contact), pigeons were allowed to continue digging in that pot but could not switch to the other side pot. If they tried to switch, the side covers were closed. The correct side pot (matching gravel for the matching group or nonmatching gravel for the oddity group) contained 20 seeds (same number and type as the sample reward). Incorrect choices were followed by a 30 -s time-out with the unaltered tray remaining in view with the transparent pot covers closed. A correction trial was then conducted. On correction trials, the center-pot cover was opened, and the pigeon was required to make contact with the sample gravel remaining in the pot (or with the empty pot). The side-pot covers were then opened, and another choice was made. Correction trials were repeated until the pigeon made the correct choice. Only performance on the first trial figured in the analyses of this article.

Daily experimental sessions contained eight trials, the same number of trials previously used (Wright \& Delius, 1994). Birds in the samplereinforcement subgroups received 16 reinforcements of 20 seeds for a total of 17.8 grams. There were 12 unique trial types with three training stimuli. Two randomized blocks of these 12 trial types were conducted over three consecutive eight-trial sessions. Trial sequences were different for different subject pairs, but they were the same for both pigeons of each pair. Pigeons were trained until they were accurate on seven out of eight trials on two consecutive sessions.

\section{Results and Discussion}

Acquisition of matching and oddity is shown in Figure 1 for individual subjects. Sample reward enhanced acquisition of matching and retarded acquisition of oddity. The oddity group without sample reward learned faster than any other group, replicating the oddity advantage shown in previous studies. These differences in matching and oddity acquisition were supported by a three-way analysis of variance (ANOVA) showing significant effects of sessions, $F(24,56)=6.8, p<.0001$; matching versus oddity, $F(1$, $56)=13.7, p<.0001$; and sample reward, $F(1,56)=4.3, p<$ .05 . Sample reward had opposite effects on matching and oddity as shown by a significant Matching-Oddity $\times$ Sample-Reward interaction, $F(1,56)=61.0, p<.0001$.

A sessions-to-criterion analysis also supports these acquisition differences. Sessions to criterion for matching were reduced by sample reward (15.0 vs. 24.5 sessions with SEMs of 5.0 and 0.5 , respectively), whereas sessions to criterion for oddity were increased by sample reward ( 18.5 vs. 7.5 sessions with $S E M$ s of 2.5 and 1.5, respectively). A two-way ANOVA on the number of sessions to criterion showed a significant Matching-Oddity $x$ Sample-Reward interaction, $F(1,4)=12.5, p<.03$.

Differences among groups are perhaps most clearly shown by the mean acquisition functions (for sessions in which both pigeons of each group participated) in Figure 2. Analyses of these functions were conducted to verify the order effects apparent in this figure, for example, whether acquisition by the matching group with 
ODDITY

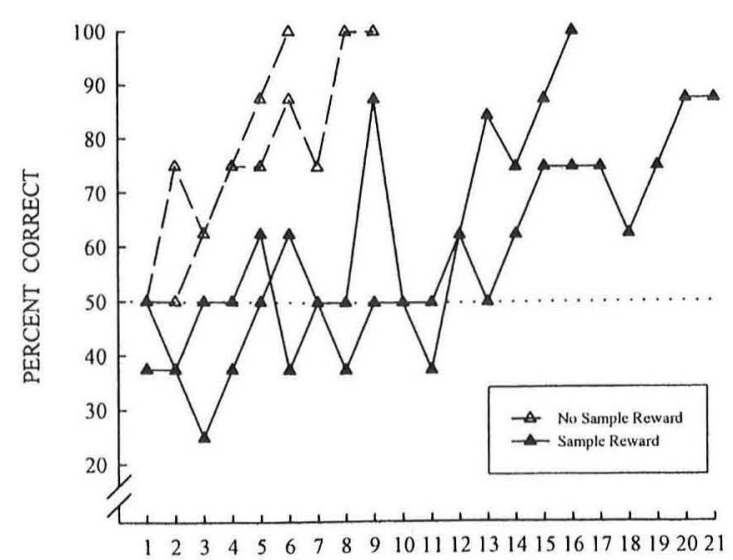

MATCHING

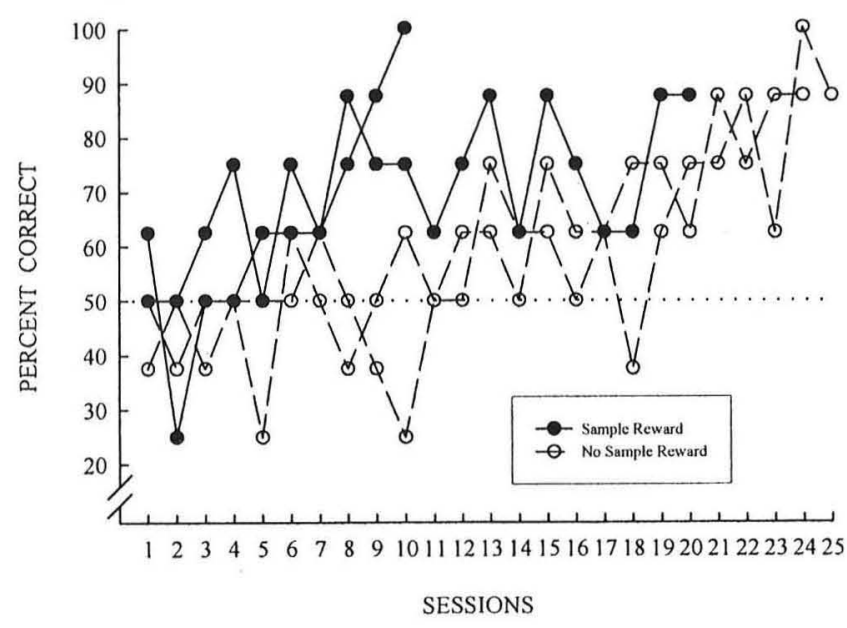

Figure 1. Acquisition by 4 pigeons in an oddity-from-sample graveldigging task (top panel) and by 4 pigeons in a matching-to-sample graveldigging task (bottom panel) in Experiment 1. Solid points are for pigeons receiving grain reward buried in the sample pot, and open points are for pigeons not receiving grain reward in the sample pot. The dotted line shows chance performance at $50 \%$ correct.

sample reward was statistically different (i.e., more rapid) from acquisition by the oddity group with sample reward. An ANOVA conducted on the linear regressions of these functions (correlations of $.98, .88, .83$, and .86 from the left) showed a significant difference among slopes, $F(3,48)=41.0, p<.001$ (Sokal \& Rohlf, 1969, p. 448). Post hoc comparisons (Simultaneous Test Procedure, Sokal \& Rohlf, 1969, p. 457) showed that each successive pair of functions differed significantly, $p \mathrm{~s}<.001$. These results confirm that oddity without sample reward was learned the fastest, followed by matching with sample reward, oddity with sample reward, and matching without sample reward. Interestingly, the worst or slowest condition-matching without sample reward-is the most frequently conducted procedure with pigeons.

No sample reward is the typical procedure for training pigeons in matching and oddity tasks and is the typical procedure for demonstrating the OPE. The most common measure of the OPE is shown by the oddity group without sample reward learning most rapidly in Figure 2. With sample reward (solid points), oddity acquisition is retarded, and matching acquisition is enhanced. The oddity group with sample reward (solid triangles) began acquisition similar to what would be expected from a matching group that might show an initial OPE. Performance began somewhat below chance performance and stayed roughly at chance performance for 11 sessions - almost twice the number of sessions for learning to have been completed by the oddity group trained without sample reward. By contrast, pigeons in the matching task given sample reward (solid circles) learned more rapidly than those not given sample reward and even more rapidly than those in the oddity task with sample reward. Thus, contrary to the hypothesis that subjects come to the laboratory with a predetermined OPE, reward for sample responding produced an acquisition advantage for matching over oddity in this gravel-digging task.

\section{Experiment 2}

The purpose of Experiment 2 was to determine whether or not the pigeons in Experiment 1 had learned the general rule of matching or oddity, and if so, whether there would be differences among the groups in the degree of concept learning. Unlike previous experiments with gravel-digging procedures (e.g., Wright \& Delius, 1994), the present study used three instead of two training stimuli and found large acquisition differences that depended on the task (matching vs. oddity) and sample reward. These considerable acquisition differences might in turn be indicative of differences in how these tasks were learned and the degree of concept learning.

\section{Method}

After acquisition, the pigeons were tested for their transfer of matching or oddity performance with gravels of different colors and gravels of different textures. Each bird was tested for transfer immediately upon acquiring its task. After the first bird of each pair completed Experiment 2, this bird was maintained on regular training so that the context of alternating trials between the 2 pigeons would be maintained while its partner finished Experiment 2.

\section{Subjects and Apparatus}

The subjects and apparatus were the same as those in Experiment 1. Figure 3 shows the training and transfer gravels. The first 3 gravels are the training gravels, the next 16 gravels are the color-transfer gravels, and the last 16 gravels are the texture-transfer gravels. Colors of the transfer gravels were different from the training gravels. Each successive pair of gravels was always tested together, as shown in Table 1. One member of each pair was the sample (and matching comparison), and the other member was the nonmatching comparison. Each color-transfer pair had the same texture, but texture differed across pairs and differed from the training gravels' textures. For each texture-transfer pair, the color of each gravel pair was the same and differed from training or color-transfer gravels. (Differences were more apparent with real gravels than with pictures.)

\section{Procedure}

Two transfer trials were intermixed (quasi-randomly on Trials 2 to 7 ) with six training trials. The contingencies of reinforcement for sample and choice responses were the same as those on training trials, but with no 


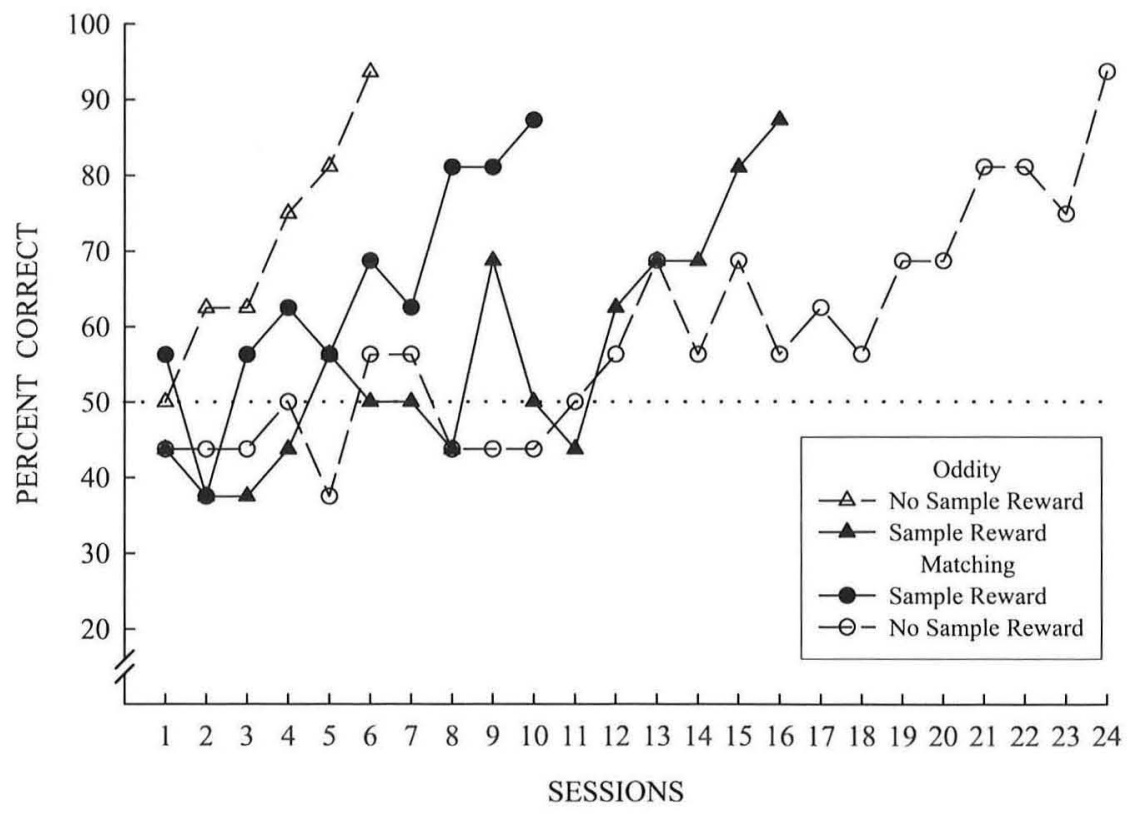

Figure 2. Mean acquisition for the four groups in Experiment 1 for sessions in which both pigeons of each group participated. The dotted line shows chance performance at $50 \%$ correct.

correction trials. There were two blocks of four sessions of color transfer followed by two blocks of four sessions of texture transfer. All eight pairs of transfer gravels (color or texture) were tested in each block. Transfer testing was then repeated for two more blocks of color transfer followed by two more blocks of texture transfer. In the first block of color transfer, the first gravel of each pair shown in Figure 3 was the sample (and matching comparison). The roles (sample and incorrect comparison) of each pair were reversed in the second block and alternated back and forth for each subsequent block. The order of testing stimulus pairs and their positions in test sessions varied from block to block. The same scheme was used for testing the texture-transfer stimuli.

\section{Results and Discussion}

Transfer to color and texture is shown in Figure 4. Baseline performance was pooled over the color and texture tests because a paired $t$ test showed that the baseline performance did not differ between these tests, $t(7)=1.44, p>.19$. The group showing the best transfer was the oddity group without sample reward. This group showed both excellent color transfer and texture transfer. Paired $t$ tests on the individual transfer results showed that neither color nor texture transfer differed significantly from that subject's baseline performance during each transfer test block, $t \mathrm{~s}(3)<1.7$, $p \mathrm{~s}>$.19. Partial texture transfer was shown by the matching group with sample reward. One pigeon showed texture transfer $(M=$ $78.0 \%$ correct with $87.5 \%$ correct on the first test block) that was significantly different from chance, $t(3)=9.0, p<.003$; the other pigeon showed texture transfer $(M=62.5 \%$ correct with $75.0 \%$ correct on the first test block) that approached statistical significance, $t(3)=2.6, p=.08$. Neither pigeon showed color transfer significantly different from chance performance, $t \mathrm{~s}\langle 1.6, p \mathrm{~s}\rangle$ .20. None of the other pigeons in the other two groups (the matching group without sample reward and the oddity group with sample reward) showed transfer to either color or texture; paired $t$ tests showed that for each subject, color and texture transfer was not different from chance performance, $t \mathrm{~s}(3)<1.6, p \mathrm{~s}>.20$.

Pigeons in this experiment could conceivably have learned something about the test stimuli despite alternating the roles of the stimuli (sample vs. nonmatching comparison) during the four presentations of each stimulus pair in Figure 3. It should be noted that this possibility of learning contaminating transfer performance cannot be completely eliminated by extinction or rewarding either choice response and can be accomplished only by using trialunique transfer stimuli (Katz \& Wright, in press; Katz, Wright, \& Bachevalier, 2002; Wright, 1991; Wright, 1997; Wright, Cook, Rivera, Sands, \& Delius, 1988; Wright, Rivera, Katz, \& Bachevalier, 2003). Unfortunately, sufficient gravel stimuli for trial-unique tests in this study could not be obtained.

Nevertheless, additional evidence supports the validity of the high level of transfer performance by the oddity group without sample reward. Any learning due to a history of reinforcement would be shown as an increasing trend over the four transfer-test blocks with color and texture stimuli. But there were no performance trends as shown by separate repeated measures ANOVAs over the four color-transfer blocks, $F(1,3)=2.5, p>.2$, and over the four texture-transfer blocks, $F(1,3)=1.3, p>$.4. Also, first-trial performance (i.e., first test block) was $87.5 \%$ correct for color and $75.0 \%$ correct for texture, which, in each case, was well within the range of the respective mean performances of $84.4 \%$ and $81.3 \%$ as shown by the error bars in Figure 4. Moreover, any claim that rapid learning might account for the good transfer by the oddity group without sample reward would have to explain why 


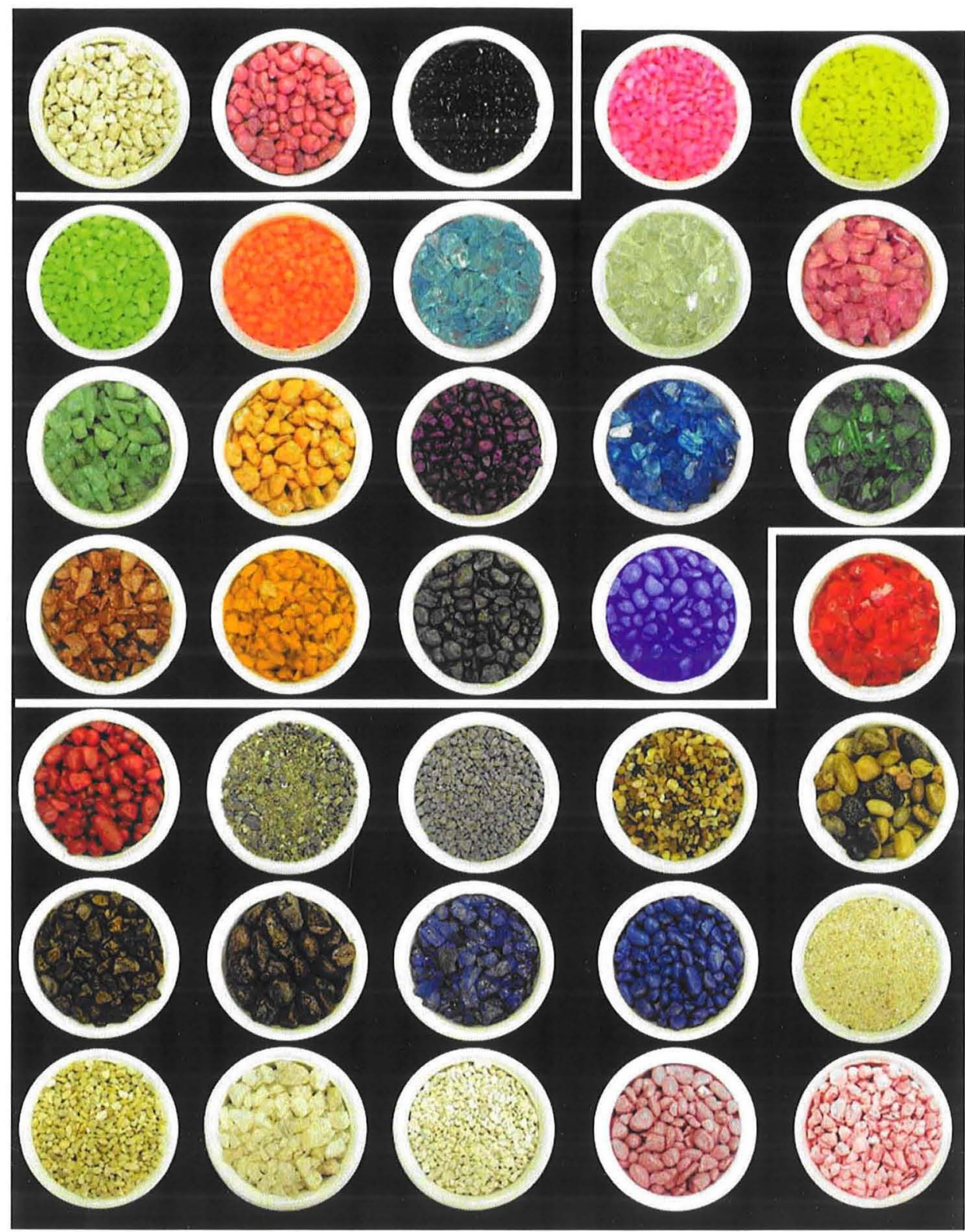

Figure 3. Gravel stimuli in pots used in Experiments 1 and 2. The first 3 gravel stimuli were the training stimuli. The next 16 gravel stimuli were the color-transfer stimuli. The last 16 gravel stimuli were the texture-transfer stimuli.

pigeons in two other groups (the matching group without sample reward and the oddity group with sample reward) showed no learning at all with these transfer stimuli.

Overall (i.e., across the four groups), transfer is shown to vary directly with the rate of learning. This finding means that a task that is rapidly learned will be more readily generalized to novel stimuli (an abstract concept). No one (that we know of) has ever shown such a result before. It does, however, make perfect sense: The easier that some task is learned, the more likely it is that subjects will notice relationships between stimuli and the better they will transfer to novel stimuli. The reason we were able to show this relationship between acquisition and transfer was that sample reward dissociated matching versus oddity learning, which produced an orderly sequence of acquisition rates with the same stimuli, the same responses, and the same choices. Moreover, we were able to show that when partial transfer occurred (matching group with sample reward) it was to texture, not color. This result suggests that a feature other than color (texture in this case) may be dominant in gaining control over the pigeon's behavior.

\section{General Discussion}

The oddity group without sample reward showed an OPE-an effect on acquisition that has been shown for almost 5 decades of 
Table 1

Training and Transfer Gravel Stimuli

\begin{tabular}{|c|c|c|}
\hline \multirow[b]{2}{*}{ Training gravels } & \multicolumn{2}{|c|}{ Transfer gravels } \\
\hline & Color transfer & Texture transfer \\
\hline \multirow{16}{*}{$\begin{array}{l}\text { 1. Coarse white chipped coral } \\
\text { 2. Mauve Ultrastone } \\
\text { 3. Fine black volcanic stone }\end{array}$} & 4. Fluorescent pink chipped Ultrastone & 20. Red chipped glass \\
\hline & 5. Fluorescent yellow chipped Ultrastone & 21. Red Ultrastone \\
\hline & 6. Fluorescent green chipped Ultrastone & 22. Gray shell \\
\hline & 7. Fluorescent orange chipped Ultrastone & 23. Gray crushed aggregate \\
\hline & 8. Aqua chipped glass & 24. Mix chipped aggregate \\
\hline & 9. Clear chipped glass & 25. Mix Ultrastone \\
\hline & 10. Pale pink gravel crystals & 26. Brown Ultrastone \\
\hline & 11. Pale green gravel crystals & 27. Brown rock \\
\hline & 12. Gold Ultrastone & 28. Blue quartz crystal \\
\hline & 13. Purple Ultrastone & 29. Blue Ultrastone \\
\hline & 14. Blue chipped glass & 30. Beige sand \\
\hline & 15. Green chipped glass & 31. Beige crushed aggregate \\
\hline & 16. Copper quartz crystal & 32. White quartz crystal \\
\hline & 17. Mustard quartz crystal & 33. White crushed aggregate \\
\hline & 18. Slate gray Ultrastone & 34. Pastel pink Ultrastone \\
\hline & 19. Royal blue Ultrastone & 35. Pastel pink chipped aggregate \\
\hline
\end{tabular}

Note. Numbers correspond to positions beginning at the top left of Figure 3 and moving from left to right.

matching and oddity research with pigeons (Ginsburg, 1957). Previously, there has been no viable explanation for what causes the OPE. Nevertheless, the OPE has been used to explain the shifted-nonshifted effect (e.g., oddity to matching vs. oddity to oddity) in the matching and oddity literature (Wilson et al., 1985a, 1985b), an effect that had previously been interpreted as evidence of pigeon abstract-concept learning (Zentall et al., 1981; Zentall \& Hogan, 1974, 1975, 1976, 1978).

Following the demonstration that the shifted-nonshifted effect resulted from the OPE, Wilson et al. (1985a) concluded, "When

ODDITY

MATCHING

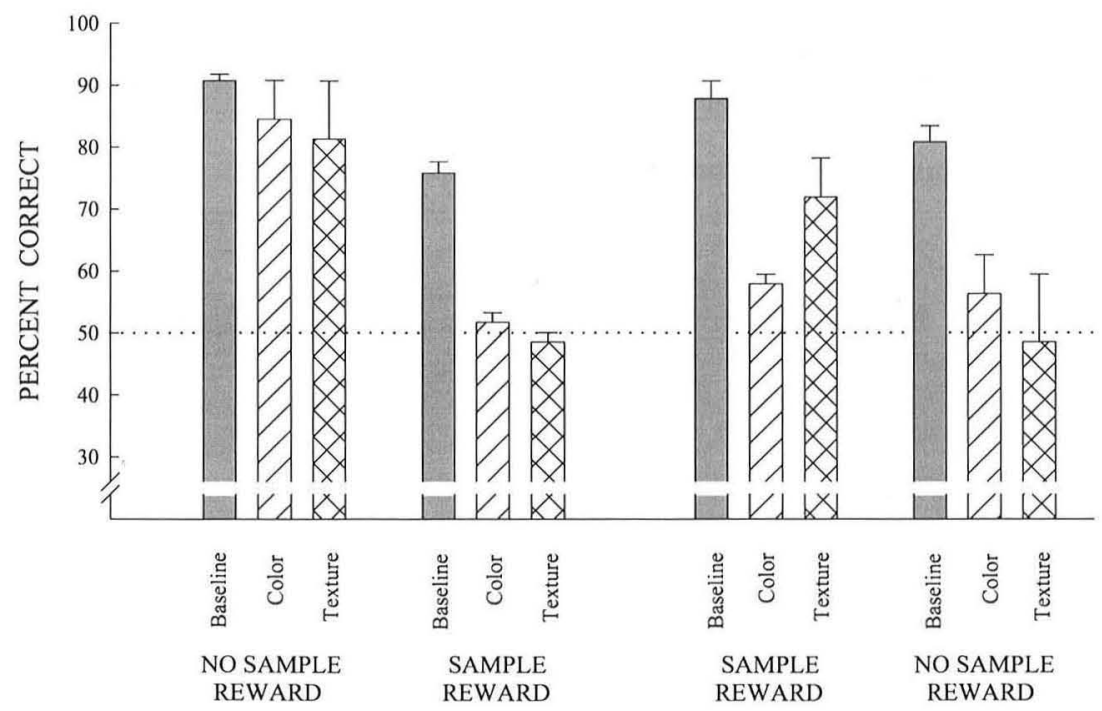

Figure 4. Mean color and texture transfer in Experiment 2 for the four groups trained in Experiment 1. Baseline performance is the mean training trial performance during all transfer testing. Error bars are the standard error of the mean. The dotted line shows chance performance at $50 \%$ correct. 
pigeons are transferred to novel stimuli, they characteristically revert to their oddity preference, and so it seems that even birds that have previously learned to ignore their preference may revert to it with novel stimuli" (p. 308). This statement implies that any novel-stimulus transfer may be due to an inadvertent OPE rather than a so-called higher order or relational comparison between the sample stimulus and the correct comparison. Although the OPE could conceivably account for some small amount of positive transfer to novel stimuli, the OPE is much too small of an effect to account for the high level of transfer by the oddity group without sample reward shown here. The magnitude of the OPE on the initial session was less than a $10 \%$ advantage. Although this slight advantage may have a profound and cumulative effect on acquisition, it would not be sufficient to account for the $83 \%$ correct transfer by the oddity group without sample reward. This group was tested with novel stimuli that differed in both color and texture from the training stimuli. Separate tests of color and texture differences (sample vs. comparison differences) showed no significant difference from their respective baseline performance-a result that is many levels above simply showing a difference from chance performance. By showing that novel-stimulus transfer was equivalent to training performance, we can claim that these birds have fully learned the abstract concept of oddity (Wright, 1991). Although previous studies have shown better than chance oddity transfer in pigeons (e.g., Lombardi, Fachinelli, \& Delius, 1984), this article is the first example in the literature demonstrating that pigeons can fully learn an abstract concept of oddity, and one of the few examples of pigeons fully learning any abstract concept. Other examples would be pigeons fully learning the abstract concept of matching-to-sample with video cartoons presented from the chamber floor (Wright, 1997; Wright et al., 1988) and pigeons fully learning an abstract concept of same/different with digitized travel slides (Katz \& Wright, in press).

The present study showed that the OPE was a major contributing factor to acquisition for the oddity group without sample reward. When sample reward was given for other pigeons in an oddity task, they took more than twice as long (18.5 vs. 7.5 sessions) to learn the task. Moreover, there was a similar but opposite effect in the matching task. When reward was given for sample responses in the matching task, pigeons learned the task faster (15.0 vs. 24.5 sessions) than those without sample reward did. Sample reward considerably enhanced learning of the matching task and even reversed the OPE finding by showing somewhat faster learning for matching than oddity with sample reward. These general relationships of sample reward on matching and oddity acquisition would be expected to be found in other settings (e.g., three-key Skinner boxes) and with other species, but the relative magnitude of these opposing effects (OPE vs. sample reward) will necessarily vary. In one monkey study, for example, there was a large OPE (80\% and $40 \%$ for oddity and matching groups, respectively, with trial-unique stimuli over the first 40 trials) despite a (highly desired) grape reward for a sample response (Mishkin \& Delacour, 1975). But relative changes in these relationships cannot be assessed in the Mishkin and Delacour (1975) study because the comparative conditions of no sample reward were not conducted.

The OPE may have aspects in common with the novelty preference in visual paired-comparison (VPC) tasks (cf., Delius, 1994). In a typical VPC task, monkeys or children look at a particular picture (for a sufficient time to habituate) and are later presented with this same picture and a novel picture. The typical result is that they look more at the novel picture than the familiar picture (e.g., Fagan, 1974; Pascalis \& Bachevalier, 1999; Zola et al., 2000). It may be difficult to inhibit looking at and touching the novel object even with recently conditioned positive association (i.e., reward) with the sample stimulus. With VPC, like the OPE, some species (e.g., monkeys) may have a greater novelty attraction than others. Although pigeons may have less of a novelty attraction than monkeys, they too may have a viewing preference for novel stimuli. One could separate the choice response from the stimulus and possibly eliminate the OPE, but there is a catch. Subjects attend to stimuli to which they respond (e.g., Stollnitz, 1965), and if they respond to manipulanda separated from to-be-discriminated stimuli, then they often have trouble learning (e.g., Harrison, Iversen, \& Pratt, 1977).

Despite the positive effects on attention of responding to the stimuli, it is clear that (judicious) manipulations of sample reward can also enhance acquisition. For the matching group with sample reward in the present study, pecks to the sample gravel were reinforced, and this positive association apparently carried over to the comparison choice where the correct choice was the same gravel stimulus as the gravel just pecked (and reinforced) in the sample pot. But this was the incorrect choice for oddity pigeons with sample reward. By contrast, oddity pigeons with no sample reward did not have this just-completed positive association from reinforced responses to the sample gravel. Indeed, if anything, they had a negative association with pecking the sample gravel due to extinction, in other words, no reward for these sample responsespossibly an unfulfilled expectation. The expectation early in acquisition may be that all pots of gravel have a big cache of grain at the bottom. This negative association from extinction (unfulfilled expectation) of digging in the sample pot of gravel may create a tendency to choose a gravel stimulus other than the sample gravel. The same logic applies to the matching groups, but with their correct and incorrect choices reversed. For the matching groups, negative associations from extinction of sample digging responses would be expected to create tendencies to make errors.

Such positive or negative associations with the sample stimuli are, of course, stimulus-specific associations. These stimulusspecific associations produce response tendencies (i.e., tendencies to peck the stimulus for which responding was just rewarded over some stimulus with a more distant-past reward history). Such tendencies early in learning may be capable of producing large snowball effects on acquisition, eventually resulting in large acquisition differences like those shown in Figures 1 and 2. For example, if a subject has even a slight tendency to make the correct choice, then this tendency will be further strengthened by the reward that follows. On the other hand, groups with associative tendencies that favor making incorrect responses (the matching group without sample reward and the oddity group with sample reward) will have two learning strikes against them. They need to inhibit this tendency to choose the incorrect comparison, and they need to learn to make a choice that is counter to this tendency. This battle against tendencies may be fought throughout acquisition. It is interesting that the degree of transfer for all the groups in this experiment is roughly ordered according to the rate of acquisition. Those groups that learned their task more easily may have more readily noticed relationships between the sample and comparison stimuli, associated these noticed relationships with reward, and 
employed relational rules (strategies) accordingly when presented with novel stimuli.

\section{References}

Berryman, R., Cumming, W. W., Cohen, L. R., \& Johnson, D. F. (1965). Acquisition and transfer of simultaneous oddity. Psychological Reports, 17, 767-775

Carter, D. E., \& Werner, T. J. (1978). Complex learning and information processing by pigeons: A critical analysis. Journal of the Experimental Analysis of Behavior, 29, 565-601.

Cumming, W. W., \& Berryman, R. (1965). The complex discriminated operant: Studies of matching-to-sample and related problems. In D. I. Mostofsky (Ed.), Stimulus generalization (pp. 284-330). Stanford, CA: Stanford University Press.

Davenport, R. D., \& Menzel, E. W., Jr. (1960). Oddity preference in the chimpanzee. Psychological Reports, 7, 523-526.

Delius, J. D. (1994). Comparative cognition of identity. In P. Bertleson, P. Eelen, \& G. d'Ydewalle (Eds.), International perspectives on psychological science (Vol. 1, pp. 25-40). Hillsdale, NJ: Erlbaum.

Fagan, J. F. (1974). Infant recognition memory: The effects of length of familiarization and type of discrimination task. Child Development, 45 , 351-356.

Ginsburg, N. (1957). Matching in pigeons. Journal of Comparative and Physiological Psychology, 50, 261-263.

Harrison, J. M., Iversen, S. D., \& Pratt, S. R. (1977). Control of responding by location of auditory stimuli: Adjacency of sound and response. Journal of the Experimental Analysis of Behavior, 28, 243-251.

Katz, J. S., \& Wright, A. A. (in press). Same/different concept learning by pigeons. Journal of Experimental Psychology: Animal Behavior Processes.

Katz, J. S., Wright, A. A., \& Bachevalier, J. (2002). Mechanisms of same/different abstract-concept learning by rhesus monkeys. Journal of Experimental Psychology: Animal Behavior Processes, 28, 358-368.

Lombardi, C. M., Fachinelli, C. C., \& Delius, J. D. (1984). Oddity of visual patterns conceptualized by pigeons. Animal Learning \& Behavior, 12, 2-6.

Mishkin, M., \& Delacour, J. (1975). An analysis of short-term visual memory in the monkey. Journal of Experimental Psychology: Animal Behavior Processes, 1, 326-334.

Pascalis, O., \& Bachevalier, J. (1999). Nonselective neonatal hippocampal lesions impair visual recognition memory when assessed by pairedcomparison task but not by delayed nonmatching-to-sample task. Hippocampus, 9, 609-616.

Siemann, M., Delius, J. D., \& Wright, A. A. (1996). Transitive responding in pigeons: Influences of stimulus frequency and reinforcement history. Behavioural Processes, 37, 185-195.

Sokal, R. R., \& Rohlf, F. J. (1969). Biometry. San Francisco: Freeman.
Stollnitz, F. (1965). Spatial variables, observing responses, and discrimination learning sets. Psychological Review, 72, 247-261.

Wilson, B., Mackintosh, N. J., \& Boakes, R. A. (1985a). Matching and oddity learning in the pigeon: Transfer effects and the absence of relational learning. Quarterly Journal of Experimental Psychology: Comparative \& Physiological Psychology, 37B, 295-311.

Wilson, B., Mackintosh, N. J., \& Boakes, R. A. (1985b). Transfer of relational rules in matching and oddity learning by pigeons and corvids. Quarterly Journal of Experimental Psychology: Comparative \& Physiological Psychology, 37B, 313-332.

Wright, A. A. (1991). Concept learning by monkeys and pigeons. In M. Corballis, K. G. White, \& W. Abraham (Eds.), Memory mechanisms: A tribute to G. V. Goddard (pp. 247-273). Hillsdale, NJ: Erlbaum.

Wright, A. A. (1997). Concept learning and learning strategies. Psychological Science, 8, 119-123.

Wright, A. A., Cook, R. G., Rivera, J. J., Sands, S. F., \& Delius, J. D. (1988). Concept learning by pigeons: Matching-to-sample with trialunique video picture stimuli. Animal Learning and Behavior, 16, 436444.

Wright, A. A., \& Delius, J. D. (1994). Scratch and match: Pigeons learn matching and oddity with gravel stimuli. Journal of Experimental Psychology: Animal Behavior Processes, 20, 108-112.

Wright, A. A., Rivera, J. J., Katz, J. S., \& Bachevalier, J. (2003). Abstractconcept learning and list-memory processing by capuchin and rhesus monkeys. Journal of Experimental Psychology: Animal Behavior Processes, 29, 184-198.

Zentall, T. R., Edwards, C. A., Moore, B. S., \& Hogan, D. E. (1981). Identity: The basis for both matching and oddity learning in pigeons. Journal of Experimental Psychology: Animal Behavior Processes, 7, $70-86$.

Zentall, T. R., \& Hogan, D. E. (1974). Abstract concept learning in the pigeon. Journal of Experimental Psychology, 102, 393-398.

Zentall, T. R., \& Hogan, D. E. (1975). Concept learning in the pigeon: Transfer to new matching and nonmatching stimuli. American Journal of Psychology, 88, 233-244.

Zentall, T. R., \& Hogan, D. E. (1976, January 30). Pigeons can learn identity or difference, or both. Science, 191, 408-409.

Zentall, T. R., \& Hogan, D. E. (1978). Same/different concept learning in the pigeon: The effect of negative instance and prior adaptation to transfer stimuli. Journal of the Experimental Analysis of Behavior, 30, 177-186.

Zola, S. M., Squire, L. R., Teng, E., Stefanacci, L., Buffalo, E. A., \& Clark, L. R. (2000). Impaired recognition memory in monkeys after damage limited to the hippocampal region. Journal of Neuroscience, 20, 451463. 